
allemande

45-2 | 2013

Images et discours de la nation

\title{
Pour ne plus être le miroir de l'Allemagne
}

L'émancipation de la photographie dans la seconde moitié des années cinquante

\section{Anaïs Feyeux}

\section{(2) OpenEdition}

1 Journals

Édition électronique

URL : https://journals.openedition.org/allemagne/1516

DOI : $10.4000 / a l l e m a g n e .1516$

ISSN : 2605-7913

Éditeur

Société d'études allemandes

\section{Édition imprimée}

Date de publication : 30 décembre 2013

Pagination : 339-356

ISSN : 0035-0974

\section{Référence électronique}

Anaïs Feyeux, «Pour ne plus être le miroir de l'Allemagne », Revue d'Allemagne et des pays de langue allemande [En ligne], 45-2 | 2013, mis en ligne le 29 juillet 2019, consulté le 20 mai 2021. URL : http:// journals.openedition.org/allemagne/1516 ; DOI : https://doi.org/10.4000/allemagne.1516 


\section{Pour ne plus être le miroir de l'Allemagne L'émancipation de la photographie dans la seconde moitié des années cinquante}

\section{- Anaïs Feyeux*}

Sortir d'un totalitarisme ne fut pas chose aisée dans les zones occidentales. Si la zone d'occupation russe a un ennemi tout trouvé en la figure du capitalisme américain et peut fédérer son caractère unitaire autour de ce rejet, cela est plus difficile à l'Ouest. Au-delà de l'idée usuelle d'une continuité des élites nazies - que l'analyse poussée pondère, mais qui démontre cependant combien il n'y eut pas de bannissement en bloc de la période totalitaire -, le discours historique a fait de l'Allemagne de l'Ouest un pays construit à la suite de regroupements économiques ${ }^{(1)}$. Comme l'explique Heinrich Böll dans un entretien de 1976, la RFA d'alors s'apparente plus à un État qu'à une patrie ${ }^{(2)}$. À la Kulturnation du XIX ${ }^{\mathrm{e}}$ siècle $^{(3)}$ répond un pays par défaut dans lequel l'art et la culture ne semblent plus revêtir le rôle officiel qui avait été le leur jusque-là ${ }^{(4)}$. Ce changement

* Docteur en histoire de l'art, Université Paris 1 Panthéon-Sorbonne.

1 La naissance de la Bizone le $1^{\text {er }}$ janvier 1947 est consécutive au regroupement économique des zones britannique et américaine, à laquelle la France s'allie en août 1948 pour former la Trizone. Voir « Joint Statement by Secretary of State Byrnes and Foreign Secretary Bevin (3 December 1946) ", in: The Department of State, Occupation of Germany, Policy and Progress 1945-46, Washington, U.S. Government Printing Office, 1947, p. 169-174.

2 Dans cet entretien, Böll parle de « destruction de la patrie par les nazis », Heinrich BöLl, Une mémoire allemande, Entretiens avec René Wintzen, Paris, Éd. du Seuil (coll. Traversée du siècle), 1978, p. 34.

3 C'est l'historien Friedrich Meinecke qui, en 1908, a popularisé cette expression. Voir Friedrich Meinecke, Weltbürgertum und Nationalstaat. Studien zur Genesis des deutschen Nationalstaats (Cosmopolitisme et État national. Études sur la génèse de l'État national allemand), Munich/Berlin, R. Oldenbourg, 1908.

4 Les intellectuels et les artistes - dont les écrivains du Groupe 47 - n’ont plus de véritable engagement politique. Hansgerd Schulte écrit à propos des écrivains d'alors: «Il y avait bien une tendance politique commune, mais sans aucune action concrète. On se situait à gauche, mais dans "une gauche sans patrie", c'est-à-dire sans engagement dans aucun parti politique », Hansgerd SCHulTE, « Les écrivains 
est perçu comme la conséquence de la perversion de la culture par le régime national-socialiste ${ }^{(5)}$. Il découle aussi d'une République fédérale d'Allemagne qui rechigne à placer la culture au cœur de sa naissance. L'anecdote que relate Hansgerd Schulte est un exemple révélateur: Ludwig Erhard, le père du miracle économique, use à propos des écrivains Heinrich Böll, Günter Grass ou Martin Walser, du mot de Pinscher, « roquets braillards mais inoffensifs » afin de mettre en valeur leur peu d'importance dans la vie publique ${ }^{(6)}$. Écarté du discours public, le monde culturel devient une simple catégorie du champ social ${ }^{(7)}$.

Ce n'est pas le cas de la photographie. Non intégrée aux beaux-arts sous le nationalsocialisme, elle profite après-guerre de ce statut particulier et ne connaît pas le discrédit des disciplines artistiques auprès des instances dirigeantes. Il faut dire que durant cette décennie, la photographie est avant tout un bien de consommation de masse. Grâce à sa multitude d'utilisations, elle est incontournable et revendique sa présence dans toutes les sphères de la société allemande. L'acquisition d'un appareil photo est facile, et ce dans les couches sociales les plus diverses. Le médium trouve un engouement sans pareil dans le pays. Il est, par sa place singulière en marge du champ de l'art et de ses enjeux, l'objet d'une grande attention de la part des mondes politique et industriel - qui ensemble président aux destinées de la République fédérale - et se voit associé à un projet d'envergure : celui de la reconstitution d'une société dans ce pays par défaut qu'est la République fédérale d'Allemagne. Et ce n'est pas la moindre des choses.

\section{De 1945 à 1955, la photographie à l'aune de la naissance de la République fédérale d'Allemagne}

Dans les premières années de l'après-guerre, le champ de la photographie ${ }^{(8)}$ se construit en osmose avec la naissance de la République fédérale d'Allemagne. De

et le nouvel État (1945-1955) ", in: Gilbert Krebs et Gérard Schneilin (dir.), L’Allemagne 1945-1955, De la capitulation à la division, Paris, Publications de l'Institut d'Allemand/Université de la Sorbonne Nouvelle, 1995, p. 289-298, ici p. 292.

5 Lionel Richard, Le nazisme et la culture, Bruxelles, Éditions Complexe, 2006.

6 H. Schulte, «Les écrivains et le nouvel État (1945-1955)» (note 4), p. 289.

7 Auparavant élément constitutif de la nation (voir la dénomination de Kulturnation), le monde culturel prend après 1945 une position tout autre dans laquelle il n'est plus qu'une sous-catégorie de l'économie tertiaire. Il n'adopte plus une posture d'aplomb qui prédispose à l'identité de la nation, mais devient un élément du champ social au même titre que l'industrie chimique ou l'automobile. Cette séparation entre art et politique modifie complètement la relation des artistes à l'État et à la société.

8 Comme s'est appliquée à le montrer une partie importante de la sociologie de l'art, les pans esthétique, politique et sociologique sont liés et déterminent plusieurs cercles d'études de la pratique artistique: le cercle du « champ» (Pierre Bourdieu), celui du « monde » (Howard Becker), celui de la «configuration» (Nathalie Heinich). Selon le cercle choisi par le sociologue, l'artiste est dépendant soit de son domaine d'activité, soit des interactions nécessaires à la création et la diffusion de son œuvre, soit du réseau dans lequel il s’inscrit de façon élargie. Ces trois approches abordent des problématiques distinctes: celle de l'affirmation d'une spécificité fonctionnelle, voire d'une autonomie du champ étudié (Bourdieu), celle d'une interdépendance entre production et diffusion amenant à la création de conventions de fonctionnement (Becker) ou celle de la mise en place d'un socle de référents communs tenant plus aux évolutions des idées qu'à des changements matériels (Heinich). Ces trois pans de la sociologie de l'art créent une vision d'ensemble de la pratique artistique: de la création concrète à la répercussion symbolique, du cadre restreint au domaine culturel au sens large, 
l'occupation par les puissances alliées, où son utilisation est avant tout politique, au miracle économique des années cinquante, tout dans le domaine photographique fait du médium le miroir à petite échelle de la construction du nouvel État. Se font jour trois grands axes de similitude avec l'histoire plus générale de l'Allemagne de l'Ouest: la reconstruction qui s'opère à l'échelle nationale comme à un niveau plus régional, l'union ou plutôt la fédération en pôle de compétence, et la volonté de retrouver liberté d'entreprendre et expérimentation aussi bien dans le domaine des industries que dans ceux de la culture et de la pensée.

\section{Le chemin de croix de la reconstruction}

Plus qu'une réaction à l'utilisation de la photographie par la propagande nationalesocialiste, la place du médium dans l'après-Seconde Guerre mondiale est une réponse à la politique de sape opérée par les Alliés entre 1945 et 1947. Durant plus de deux ans, les forces d'occupation lèvent les brevets des industries allemandes, démantèlent consciencieusement tous les regroupements économiques, supervisent les organes d'information et cantonnent les professionnels autochtones aux photographies de passeport. Dans le même temps, ils dressent un tableau apocalyptique de la mentalité allemande contemporaine et de l'histoire du pays à l'aide d'essais photographiques dans les grands magazines de l'époque, de livres et d'affiches placardées sur les murs des villes ${ }^{(9)}$.

Cette politique qui accuse la nation dans son ensemble des exactions du régime nazi, et ce en partie grâce au médium photographique, est telle que les photographes allemands restés au pays se sentent dépossédés de leur destin. En 1947, le magazine photographique Foto-Spiegel regrette, par exemple, le manque d'images produites par les Allemands eux-mêmes et les incite à reprendre leur place: «Ainsi les générations futures auront-elles à créer leur connaissance visuelle d'époques cruciales de l'Allemagne principalement à partir des photos des correspondants de guerre alliés. [...] Lentement seulement, les premiers photographes allemands ont recommencé à consigner notre temps en image ${ }^{(10)}$. Il convient d'offrir de l'histoire contemporaine allemande une vision moins univoque et qui inculpe non la nation allemande, mais l'Occident. Pour réinvestir la photographie, tenter de sauver l'économie en berne et redonner à l'Allemagne la place centrale qu'elle avait dans le développement du médium jusque-là, il faut montrer que le destin allemand est moins celui d'un particularisme que de toute nation

et c'est sous la forme d'une conjugaison des trois termes que doit s'entendre l'utilisation du terme " champ » faite dans l'article.

9 Voir entre autres à ce sujet: Jörn GLASENAPP, Die deutsche Nachkriegsfotografie, Eine mentalitätsgeschichte in Bildern, Paderborn, Wilhelm Fink, 2008, p. 75; Ute EsKiLdsEn, "Zur Entwicklung deutscher Illustrierten seit 1945 ", in: ID., Fotografie in deutschen Zeitchriften 1946-1984, Stuttgart, Institut für Auslandsbeziehungen, Ausstellungsserie Fotografie in Deutschland von 1850 bis heute, 1985, p. 4-15, ici p. 5; Christian Delage, «L'image photographique dans le procès de Nuremberg ", in: Clément Chéroux (dir.), Mémoire des camps, Photographies des camps de concentration et d'extermination nazis (1933-1999) (Cat.), Paris, Marval, 2001, p. 172-173.

10 «So werden also künftige Generationen ihre visuelle Kenntnis entscheidender Zeitabschnitte Deutschlands hauptsächlich aus den Fotos alliierter Kriegskorrespondenten zu schöpfen haben. [...] Nur langsam haben die ersten deutschen Fotografen wieder begonnen, unsere Zeit im Bild festzuhalten. » Anonyme, «Das Foto - Abbild der Zeit », Foto-Spiegel, novembre 1947, $1^{\text {re }}$ année, nº 2, p. 2. 
civilisée et que les zones occidentales ne peuvent être exclues du mouvement global de reconstruction à l'échelle européenne. La photographie joue un rôle majeur dans ce processus. Depuis la naissance du Reich bismarckien, le médium a été un des ferments de la représentation nationale et a permis à l'Allemagne d'être reconnue à l'étranger à l'égal des grandes nations occidentales qui avaient connu des révolutions industrielles plus précoces. Le processus connaît un coup d'arrêt avec la politique des Alliés dans les zones occidentales qui laisse peu de marge de manœuvre aux autochtones.

Après avoir voulu réduire l'ancien Reich à des terres arables, les Alliés font marche arrière, mais décident de conserver la mainmise sur les industries lourdes dont celles de la Ruhr. Il ne reste plus aux Allemands que les industries de pointe, réduites pourtant qu'elles sont à des entreprises de taille mineure. Les bombardements et la partition des usines entre différentes zones qui ne peuvent dialoguer entre elles portent un coup aussi dur que la levée des brevets. Même Agfa, fleuron de l'industrie photographique jusque-là, connaît des premières années difficiles après le démantèlement du cartel industriel IG Farben auquel elle appartenait ${ }^{(11)}$. Le dérèglement, par les Alliés, du fonctionnement de chaque pan de la photographie est important: modification de l'industrie, en redonnant une autonomie à chacune des entreprises; mis à bas des structures, en mettant fin à la publication des magazines nationaux-socialistes et en détruisant nombre de lieux d'expositions, et mainmise sur l'esthétique en interdisant aux photographes professionnels, dans les premières années, toute production qui ne soit pas un portrait de passeport ou une commande passée par les troupes d'occupation pour leurs journaux. À cette confiscation par les Alliés des pans professionnels de la photographie, il faut ajouter la perte des appareils durant la guerre ou leur réquisition, anéantissant autant la production amateur que professionnelle. Si quelques livres sont édités et sont des succès de librairie - dont celui, fameux, de Hermann Claasen ${ }^{(12)}$ - et si des expositions font date dans l'histoire de la photographie allemande de l'aprèsguerre $^{(13)}$, ces projets restent ponctuels et indépendants les uns des autres, démontrant que chacun, qui du photographe artistique, qui du photoreportage, tente de reconstituer les moyens de légitimation dont il use habituellement, sans penser cependant une vue d'ensemble.

11 Jean-Philippe Massoubre, L'histoire de l'IG-Farben 1905-1952, Paris, L'Harmattan, 2008, p. 97. Un procès concernant vingt-trois dirigeants de l'IG Farben se tient du 13 mai 1947 au 30 juillet 1948. Les dirigeants sont symboliquement jugés à Nuremberg, dans la même salle que les responsables politiques et militaires.

12 Hermann ClaAsen, Gesang im Feuerofen, Köln - Überreste einer alten deutschen Stadt, Introduction de Franz A. Hoyer, Mise en forme de Werner Labbé, Düsseldorf, Verlag L. Schwann, 1947, 1949 (2éd.), 1979 ( $3^{\mathrm{e}}$ éd.), 1980 (4éd.).

13 Peuvent être ici citées les expositions 1. Große Ausstellung der Stuttgarter Photographischen Gesellschaft 1947. Die Photographie 1948. Photographik, Bildbericht, Photogramm qui se tient au Landesgewerbemuseum de Stuttgart en 1948 et $2^{e}$ Foire, Exposition de la Photo et du Cinéma d'Amateur 1949/ II. Messe, Ausstellung der Photo-Kino-Industrie 1949 à la Staatliche Schule für Kunst und Handwerk de Neustadt a. d. Haardt (Saalbau) en 1949. 


\section{Le rôle central de l'industrie photographique}

L'industrie, à la suite de regroupements progressifs, tout d'abord clandestins puis rapidement institutionnalisés, offre à la photographie une seconde vie. Elle cherche à constituer une unité, chacune des entreprises encore en service étant de taille trop restreinte pour penser faire ombrage aux firmes étrangères. La réunion est une nécessité historique et économique autant qu'une sorte de " contre-pouvoir " face aux Alliés, à même de permettre de retrouver une indépendance et de redevenir une force vive. La coopération se fait en deux temps: horizontale tout d'abord grâce à l'Association des fabricants d'articles photographiques fondée en 1946, puis horizontale et verticale lorsque naît l'Apho (Arbeitsausschuß der deutschen Photowirtschaft) le 15 septembre 1948, dépassant l'idée de cartel au profit du concept de Konzern ${ }^{(14)}$.

L'Apho regroupe tous les acteurs du champ puisque ce comité de travail sur l'économie photographique allemande fondé par Bruno Uhl, alors à la tête de l'entreprise Agfa de Leverkusen, est composé des membres importants de toutes les associations économiques touchant au domaine photographique: fabricants, grossistes et négociants en matériel photographique, laboratoires ou entreprises de photocopies et même photographes ${ }^{(15)}$. D'autres associations aux intérêts proches comme les associations d'amateurs ou l'industrie du cinéma intègrent le comité de travail. Présidé naturellement par Uhl - qui supervisait déjà l'Association des fabricants d'articles photographiques, rebaptisée "Association de l'industrie photographique allemande » (Verband der Deutschen Photographischen Industrie) en $1950^{(16)}$-, l'Apho est pensé comme une chambre au sein de laquelle toutes les questions relevant de l'économie photographique doivent être soulevées et discutées.

En plus d'un regroupement économique, l'Apho devient dès sa création un organe de promotion des produits photographiques par la mise sur pied d'une politique culturelle photographique. Dès son discours du 9 janvier 1948, Uhl défend l'idée de promouvoir la photographie comme un objet culturel à même de soutenir indirectement la croissance d'une industrie photographique renaissante. Il conçoit la promotion du médium selon quatre voies: foire photographique, presse spécialisée, école de photographie et valorisation d'une photographie professionnelle à caractère esthétique. Car Uhl l'a bien compris - accentuant là un procédé déjà en germe depuis les années vingt en Allemagne: pour devenir une grande industrie aux niveaux national et international, la photographie doit représenter autre chose qu'elle-même. Le désir des membres de l'Apho est rapidement clair, il faut faire du médium le miroir de la reconstruction allemande. L'association met alors en place des campagnes publicitaires à l'échelle nationale vantant la photographie comme le hobby de la société de loisirs naissante. Elle va surtout être à la naissance de la photokina.

14 À la différence du cartel, le Konzern associe concentration verticale et concentration horizontale.

15 Bruno UHL, Erinnerungen, édition privée, 1970, p. 289 (Bibliothèque de la Deutsche Gesellschaft für Photographie - Museum Ludwig, Cologne).

16 J. Glasenapp, Die deutsche Nachkriegsfotografie (note 9), p. 148. 


\section{La photokina, centre névralgique de la renaissance photographique}

Foire photographique née à Cologne en 1950, la photokina accueille plus de 75000 visiteurs la première année et 176000 pour sa $4^{e}$ édition en $1954^{(17)}$. Faite pour promouvoir les productions industrielles ouest-allemandes ${ }^{(18)}$ et faire connaître la photographie naissante du pays, la photokina devient, par son envergure, leur plus sûre promotion tant sur le territoire national qu'à l'international. Les entreprises étrangères cherchent rapidement à avoir un stand à Cologne pour contrer la réussite ouest-allemande. Absentes la première année, elles représentent $20 \%$ des exposants l'année suivante, un tiers en 1958, pourcentage qui augmente et atteint plus de $50 \%$ au début des années soixante-dix ${ }^{(19)}$.

Parallèlement, cette foire de produits industriels et le comité Apho cherchent à relancer la photographie dans la sphère sociale. Ils organisent, au sein de la photokina, des expositions amateurs et montrent les utilisations possibles du médium dans divers corps de métier (médecine, science, justice, etc.) ${ }^{(20)}$. Cette double présence amateur/professionnel est primordiale. Elle dénote la volonté qu’ont les acteurs économiques de faire du médium un objet usuel et incontournable au sein de chaque famille. L'exposition inaugurale de la photokina de 1950 s'intitule « Celui qui photographie profite plus de la vie » et montre un couple photographiant les moments les plus anodins de son quotidien ${ }^{(21)}$.

17 "Statistiken ", in: Ulrich Pohlmann (dir.), Kultur, Technik und Kommerz: die photokina-Bilderschauen 1950-1980, Cologne, Historisches Archiv der Stadt Köln, 1990, p. 127.

18 Si une foire au cœur de ces immenses halles en bordure du Rhin est rendue possible, c'est avant tout grâce à la volonté des entreprises du secteur photographique qui désirent faire des appareils photographiques l'un des premiers biens d'exportation de l'Allemagne de l'Ouest qui commence à exporter de façon significative depuis 1948. La foire sert de lien entre les différentes entreprises du champ. De nombreuses entreprises font d'ailleurs partie du comité de travail. Voir la liste dressée en exergue du catalogue de 1950: «Ehrenausschuss », in: L. Fritz Gruber (éd.), Photo-Kino-Ausstellung, Köln 1950, vom 6. bis 14. Mai (Cat.), Cologne, Greven \& Bechtold, 1950, p. 5-6. La Frankfurter Allgemeine Zeitung, grand quotidien national, voit dans cette foire la démonstration d'« une place de premier plan dans le monde pour la technique allemande de photographie et de film ». Anonyme, " Hat der Photofreund mehr vom Leben? ", Frankfurter Allgemeine Zeitung, 12.05.1950, p. 8.

19 «Statistiken» (note 17), p. 127.

20 Bruno Uhl n'écrit pas autre chose dans un article publié dans le journal Rheinischer Merkur le jour de l'ouverture de la première édition de la photokina, citant les diverses utilisations du médium: «l'artisanat photo et la presse, les moyens de reproduction et la photocopie, le système de santé publique (le diagnostic par radiographie), la culture, les sciences et l'enseignement (la répartition des fruits des recherches, l'archivage, le matériel de documentation et de conférence, la technique cinématographique et le ciné-théâtre), l'industrie et la technique (l'enregistrement des documents techniques et commerciaux, le contrôle de matériels, l'analyse spectrale, les archives de sécurité et les cartes, la publicité et l'imagerie spécialisée), les services publics (photographies de passeport, police, criminalité, justice, mesures des territoires, plan de ville, publicité de circulation) ». ("Photohandwerk und Presse, Reproduktionswesen und Photokopie, Gesundheitswesen, (röntgendiagnostik), Kultur, Wissenschaft und Unterricht (Festlegung von Versuchsergebnissen, Archivierung, Anschauungs- und Vortragsmaterial, Kinotechnik und Kinotheater), Industrie und Technik (Registrierung technischer und kaufmännischer Unterlagen, Materialprüfung, Spektralanalyse, Sicherheitsarchive und Karteien, Werbung und Fachausbildung durch Bild und Film), Öffentlicher Dienst (Passbilder, Polizei und Kriminalistik, Rechtsprechung, Landesvermessung, Städteplanung, Verkehrswerbung) »). Bruno UHL, « Auf breitem Fundament », Rheinischer Merkur, 06.05.1950, p. 11.

21 «Wer photographiert hat mehr vom Leben». 
(Anonyme), «Vue de l'exposition inaugurale Wer photographiert hat mehr vom Leben », Photo-Kino-Ausstellung, Cologne, 1950. Copyright: Historisches Archiv der Stadt Köln.

Il convient de prouver au spectateur combien il peut prendre par la photographie une part active dans la société allemande en constitution. Cette omniprésence de la photographie, professionnelle et amateur, à tous les échelons de la société ouest-allemande en pleine construction donne l'image d'un médium fort.

Les photographes professionnels deviennent les forces motrices de ce renouveau et assoient le succès du médium dans la jeune Allemagne de l'Ouest. En 1951, ils se constituent en groupe uni autour d'une association, la Deutsche Gesellschaft für Photographie. Les acteurs du champ économique déjà présents dans l'Apho sont rejoints par des photographes professionnels mais aussi des acteurs du champ photographique élargi (directeur de société, de foire) et même une personnalité politique, le maire de Cologne ${ }^{(22)}$. Cette association qui regroupe toutes les personnes éminentes ayant trait à la photographie ouest-allemande est un tournant pour le champ photographique.

22 La DGPh se réunit pour la première fois le 15 mai 1951 à l'hôtel de ville de Cologne, sous la présidence du maire Görlinger. Cette réunion comprend vingt membres à part entière (les photographes Otto Croy, Adolf Lazi, Herbert List, Bernd Lohse, August Sander, Otto Steinert, Liselotte Strelow, Carl Strüwe, le fils du fondateur de l'entreprise Leitz Camera - Leica Ernst Leitz, le directeur de l'entreprise Leitz Camera - Leica Henri Dumur, le fondateur de l'entreprise Rollei Reinhold Heidecke, le directeur de l'usine Agfa Bruno Uhl, le directeur de la GDL (Gesellschaft deutscher Lichtbildner) Richard Gerling, l'organisateur de la photokina L. Fritz Gruber, le réalisateur et producteur Curt Oertel, l'homme politique Karl Weiss, Hans Kammerer, Walter Meidinger, Alfred Miller, Albert Narath, ainsi que six correspondants étrangers (le Hollandais General Lieuwe Evert Willem van Albada, le Français Louis Philippe Clerc, le professeur zurichois John Eggert, le Japonais Kitano, le membre de la Royal Photographic Society Kenneth G. Moreman et le conservateur de photographie au MoMA de New York Edward Steichen). Voir: "Vorgeschichte und Gründung ", in: Zehn Jahre Deutsche Gesellschaft für Photographie e. V. 1951-1961, Cologne, Deutsche Gesellschaft für Photographie, 1961, p. 9-31, ici p. 9-10. 
La $D G P h$ a deux objectifs qui font écho à la photokina: rapprocher les industries des photographes pour offrir aux premières un crédit culturel et faire connaître les photographes qui représentent une certaine modernité visuelle (Lazi, Steinert, List, Strelow, etc.) mais pâtissent du poids trop fort de l'ancienne génération ${ }^{(23)}$. Étrangement, l'industrie commence à défendre une photographie artistique qui semble au premier abord loin de ses propres préoccupations.

\section{Des propositions esthétiques en accord avec le nouveau pays}

En juillet 1949, des jeunes photographes réalisent un coup de maître en présentant des photographies expérimentales et abstraites en marge de l'exposition phare de Neustadt dont le jury avait refusé leurs travaux ${ }^{(24)}$. Déçus par ce refus d'exposer, six photographes fondent le groupe fotoform afin de se donner plus de visibilité ${ }^{(25)}$. L'exposition de leurs travaux artistiques au sein de la première édition de la photokina les fait connaître sur la scène nationale, au-delà du cercle restreint des photographes professionnels.

En effet, l'exposition est une «bombe atomique dans le tas de fumier de cette exposition » comme le souligne un critique d'art ${ }^{(26)}$. Pour la première fois, des jeunes photographes sont défendus par des dirigeants économiques qui voient dans l'esthétique qu'ils défendent la naissance d'une photographie artistique ouest-allemande spécifique au territoire et un attrait pour les cercles étrangers avec lesquels le groupe est en contact ${ }^{(27)}$. Si l'industrie soutient cette esthétique, très éloignée et de la pratique

23 Comme l'écrit L. Fritz Gruber dans un projet publicitaire rédigé en mai 1951, la $D G P h$ veut « encourager, mettre à l'épreuve et confronter à la discussion le nouveau audacieux, l'avant-gardiste, qui ne trouve, principalement pour des raisons mercantiles, aucune possibilité de représentation et de réalisation » (« das kühne Neue, das Avantgardistische, ermutigen, ausprobieren und zur Diskussion stellen, das wohl hauptsächlich aus merkantilen Crüden (sic) keine Möglichkeit der Darbietung und Verwirklichung findet»), cité in Ludger Derenthal, Bilder der Trümmer-und Aufbaujahre, Fotografie im sich teilenden Deutschland, Marburg, Jonas Verlag, 1999, p. 127.

24 Une section sans jury est créée par Wolfgang Reisewitz, qui avait été chargé, par la France, de la mise en place d'une exposition photographique accompagnant la grand-messe de l'industrie photographique de Neustadt. Elle présente les « domaines limites de la photographie ", c'est-à-dire les photographies expérimentales rejetées dont celles de Chargesheimer, Annelise Hager et Marta Hoepffner. Voir: Petra Reisewitz, 'fotoform', Fachbereich Visuelle Kommunikation, Offenbach, Hochschule für Gestaltung, juillet 1980, 11 p. (Archives photographiques du Museum Folkwang, Essen), p. 2 et Ulrich Pohlmann, "Zwischen Kultur, Technik und Kommerz: die photokina-Bilderschauen 1950-1980 ", in: ID. (dir.), Kultur, Technik und Kommerz (note 17), p. 10.

25 Wolfgang Reisewitz suggère aux photographes dont les productions ont été rejetées de se regrouper afin de soumettre solidairement leurs travaux dans les prochaines expositions et de pouvoir exercer une influence dans le milieu de la photographie. Otto Steinert, Ludwig Windstosser et Toni Schneiders font partie des fondateurs de fotoform. Le groupe est formé le 7 juillet 1949 à Neustadt. Peter Keetman et Siegfried Lauterwasser les rejoignent rapidement. Voir: P. ReIsEwitz, 'fotoform' (note 24), p. 4 et J. A. SChmoll Gen. Eisenwerth, «Die avantgardistische Gruppe 'fotoform' 1949-1958 », in: ID., 'subjektive fotografie' der deutsche Beitrag 1948-1963, Stuttgart, Institut für Auslandsbeziehungen, 1989, p. $12-23$, ici p. 15.

26 «Atombombe im Komposthaufen dieser Ausstellung ». Robert D’Hooghe, « Deutsche Photographie 1950 ", Frankfurter Allgemeine Zeitung, 23.05.1950, p. 5.

27 Symptôme de cela, la première action culturelle de la DGPh est le financement de la reprise partielle de l'exposition Subjektive Fotografie pour la Belgisches Haus (Maison belge) de Cologne du 21 au 28 octobre 1951. «Deutsche Gesellschaft für Photographie, Eine Chronologie - The German Photographic 
Otto Steinert, «Strenges Ballet », 1949 (n 65/32).

Copyright: Estate Otto Steinert, Museum Folkwang, Essener Systemhaus. amateur et des travaux de commande, c'est que le groupe et les expositions Subjektive Fotografie de 1951 et 1954 où ils montrent leurs travaux ${ }^{(28)}$ défendent une conception de la photographie qui répond en creux à ce qui peut séduire les amateurs: liberté, indépendance, importance donnée au photographe, abandon de l'utilisation politique des images. La rhétorique qui accompagne cette production visuelle répond aux préoccupations de la jeune République fédérale d'Allemagne qui souhaite entrer dans le bloc occidental.

Assise à partir de 1951 par cette série d'expositions nommées Subjektive Fotografie, la photographie artistique allemande devient l'instigatrice d'un nouveau courant photographique à l'échelle européenne. Le retour de l'Allemagne sur la scène occidentale grâce à sa photographie permet dès le début des années cinquante la naissance d'une fierté nationale, encore inenvisageable en 1948, qui ne repose plus sur une conception politique de la sphère sociale. Le domaine économique a retrouvé son autonomie par rapport à l'État, grâce à la reconnaissance à l'international de son industrie de pointe et de qualité et grâce au moteur que sont devenus les photographes allemands à l'échelle européenne.

Miroir de l'Allemagne de l'Ouest, la photographie professionnelle l'est aussi par les thèmes qu'elle traite alors. Dans la photographie de commande, ce sont trois sujets représentatifs de ces années-là qui sont abordés: l’industrie lourde - les mines encore

Society, a Chronology 1951-2001 », in: Susanne LANGe et Gerd SANDER (dir.), Zwischen Dokument und Abstraktion. Eine Auswahl aus der Sammlung der Deutschen Gesellschaft für Photographie - Entre document et abstraction, Un choix de la Collection de la Société allemande de Photographie - Between Document and Abstraction, A Selection from the German Photographic Society Collection, Cologne, August Sander Archiv/SK Stiftung Kultur, 1996, p. 280-291, ici p. 281.

28 La première exposition Subjektive Fotografie, organisée par Otto Steinert, un des membres de fotoform, se tient du 12 au 29 juillet 1951 à la Schule für Kunst und Handwerk de Sarrebruck. Exposition monumentale par sa taille - 725 travaux -, elle expose fotoform mais aussi d'autres photographes qui rendent compte des différents pans de la photographie d'après-guerre. Voir le catalogue: Subjektive fotografie, Saarbrücken 12-29.VII.1951, Exposition internationale de la photographie moderne organisée par la section photographique du centre de métiers d'Art sarrois à Sarrebruck (Neubau an der Ludwigskirche). 
objets de convoitises dans ces années-là - et l'industrie de pointe, l'Heimat si particulière à l'Allemagne avec des photographies de paysage et de villes posant ouvertement la question d'un territoire régional en lieu et place du national, et le portrait qui se penche sur la société, les ouvriers, les personnalités publiques, etc.

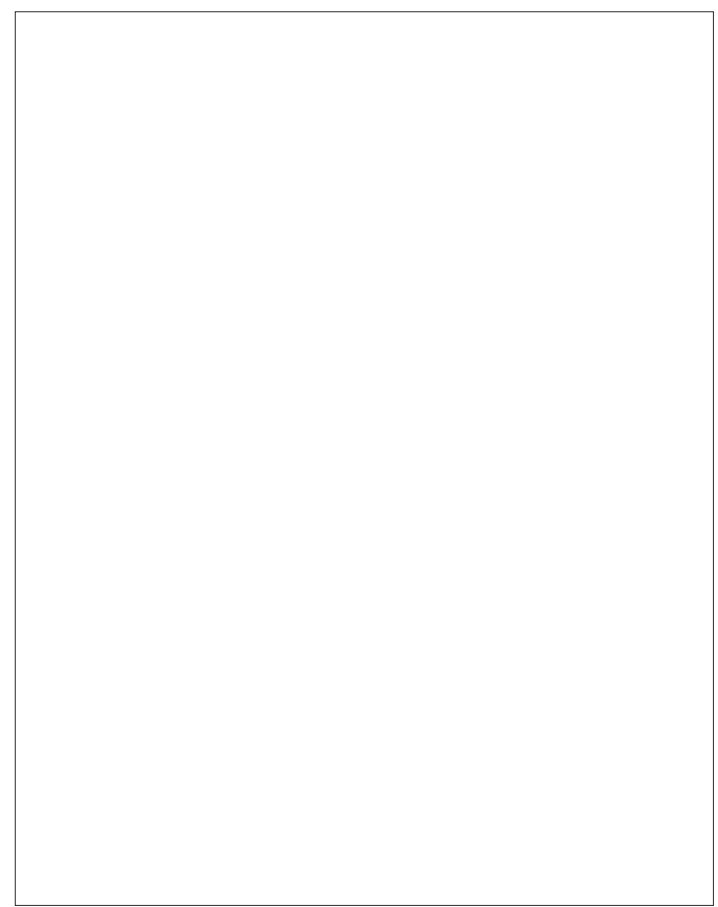

Heinrich Heidersberger, « Tankstelle Blauer See (Gas Station Blue Lake), Hannover 1953 (Architect: Dieter Oesterlen) ", 1953. Copyright: Heinrich Heidersberger, www.heidersberger.de
Dans ces premières années, le photographe est donctotalement ancré dans son époque tant par sa production visuelle qui lui permet de nouer des liens avec la scène occidentale que grâce à l'industrie nationale qui s'avère centrale dans la constitution d'un champ photographique ouest-allemand autonome et viable.

La photographie des premières années de la RFA est une représentante de la jeune RFA: reprise, à un niveau plus restreint, de l'organisation économique qui a formé la base de la nouvelle nation et de thématiques en accord avec les intérêts de l'époque. Par là même, le champ photographique perd sa force de projection utopique qu'avaient défendue les avantgardes de l'entre-deux-guerres et que souhaitait le national-socialisme dans un tout autre sens.

\section{Après 1955, une autonomisation progressive de la sphère photographique face au destin national}

De cette ascension, l'année 1955, qui marque au niveau national la fin de l'occupation occidentale, représente l'acmé et la césure, une césure en germe dès le début des années cinquante. Trois changements majeurs interdépendants se dessinent: l'internationalisation au détriment du champ photographique national, l'émergence du photoreportage qui éclipse la photographie artistique abstraite et expérimentale et les débuts d'une critique de la reconstruction allemande par les photographes, qui remettent en cause la neutralité qu'ils avaient jusque-là adoptée.

\section{L'internationalisation des enjeux}

Alors que le champ s'était jusqu'ici construit à l'échelle nationale pour reprendre place sur la scène locale et contrer la mainmise des pays étrangers dans les premières années de l'après-guerre, la seconde moitié des années cinquante marque le début 
d'une internationalisation qui va aller grandissante jusqu'au début des années soixante. Plusieurs faits marquent le point de départ de cette internationalisation: l'exposition The Family of Man née aux États-Unis, qui se déplace à Berlin et Munich en 1955, le financement de la foire photographique de Cologne par l'Unesco, et la remise en cause de l'hégémonie des firmes industrielles allemandes.

L'exposition The Family of Man, organisée par Edward Steichen, directeur du département photographique du Museum of Modern Art de New York, est monumentale, autant par le nombre d'œuvres exposées que par sa tournée internationale entre 1955 et 1964, qui touche des millions de visiteurs ${ }^{(29)}$. Berlin est la première destination hors des États-Unis ${ }^{(30)}$. Bien accueillie par le public allemand ${ }^{(31)}$ et la critique spécialisée, l'exposition marque une césure dans les propositions photographiques montrées jusque-là en Allemagne de l'Ouest. En effet, la Subjektive Fotografie, quasi hégémonique sur le terrain théorique artistique, défendait une suprématie et du photographe et de la forme visuelle. Avec The Family of Man, c'est le caractère universel du sujet photographié qui prime. Sans légende, l'exposition fait peu de cas du photographe, du lieu et du moment de la prise de vue. Les images sont regroupées en séries autour d'un sujet : la naissance, la rencontre amoureuse, la famille, ou encore la mort, ce qui semble commun aux hommes, au-delà des particularismes régionaux et temporels. Comme la Subjektive Fotografie, The Family of Man propose donc une vision universelle de la photographie. Cependant, à l'inverse de la Subjektive Fotografie, l'universalité est celle du sujet avant d'être celle d'une théorie photographique, le commissaire d'exposition devenant plus important que le photographe, dépossédé de son image. Cette nouvelle vision de la photographie remet en cause le statut hégémonique de la photographie artistique ouest-allemande en RFA. De plus, à défaut d'en être l'instigatrice comme avec la Subjektive Fotografie, l'Allemagne de l'Ouest est dans cette exposition un pays comme un autre, sans particularité( ${ }^{(32)}$. Elle n'est pas

29 Considérée comme la plus grande entreprise photographique jamais réalisée, elle réunit 503 photographies de 273 photographes professionnels ou amateurs. Plus de neuf millions de visiteurs verront l'exposition. L'exposition-mère ouvre le 26 janvier 1955 au Musée d'Art moderne de New York, mais des copies sont montrées à quatre-vingt-huit reprises dans trente-huit pays différents entre 1955 et 1962, grâce à l'appui de l'United States Information Agency (USIA - Agence d'Information des États-Unis). Voir: Eric J. SAndeen, Picturing an Exhibition, The Family of Man and the 1950s America, Albuquerque, The University of New Mexico Press, 1995, p. 95 et John Szarkowski, "The Family of Man ", in: John Elderfield (dir.), The Museum of Modern Art at Mid-Century, At Home and Abroad, New York, The Museum of Modern Art/Harry N. Abrams, "Studies in Modern Art », 4, 1994, p. 12-37, ici p. 13.

30 En Allemagne de l'Ouest, c'est une version identique à celle de New York qui est exposée. J. SzARKOwSKI, "The Family of Man» (note 29), p. 13.

31 Près de 44000 visiteurs voient l'exposition en seulement 25 jours. À Hambourg, trois ans plus tard, le succès ne se dément pas. 43308 visiteurs en un mois, dont 3520 le dernier jour. Cf. : Lettre de l'USIS à Bonn au USAI, Washington, 20 novembre 1958, archives USIA, Washington D.C., cité in Eric SANDEEN, "'The Show You See With Your Heart'. The Family of Man on Tour in the Cold War World", in: Jean BACK et Viktoria Schmidt-Lisenhoff (dir.), The Family of Man 1955-2001. Humanismus and postmoderne: Eine Revision von Edward Steichens Fotoausstellung, Humanism and Postmodernism: A Reappraisal of the Photo Exhibition by Edward Steichen, Marburg, Jonas Verlag, 2004, p. 100-121, ici p. 120.

32 Dans le choix photographique de Steichen, l'Allemagne n'est qu'un pays parmi les soixante-huit participants et est moins représentée que la France et les États-Unis. Les photographies prises sur le 
exclue, mais elle perd dans le même temps un statut d'exception qui lui avait permis après 1945 de se poser comme avant-garde.

Cela est encore plus manifeste dans l'édition 1956 de la photokina. Foire dont l'ambition première était de soutenir l'industrie ouest-allemande et de défendre la photographie nationale, elle devient une foire à vocation internationale: le Japon, grand concurrent industriel de l'Allemagne, y expose sa technologie ${ }^{(33)}$, l'Unesco finance une grande partie des expositions et le président des États-Unis Eisenhower devient, à côté du ministre ouest-allemand de l'Économie, l'autre soutien de poids de la photokina. L'Unesco comme les États-Unis usent d'un nouveau langage: " coopération internationale ", " paix dans le monde », " compréhension entre les peuples ", "fraternité » ${ }^{(34)}$. Ces mots reviennent tout au long du catalogue, même lorsque les auteurs font référence à des expositions qui différent peu des éditions précédentes, comme par exemple les photographies faites par la jeunesse du monde entier qui sont montrées à chaque foire. À cela, il faut ajouter la première exposition au sein de la photokina de la Fédération internationale de l'Art Photographique, association internationale « constituée par les fédérations nationales des divers pays " qui souhaite rassembler les peuples ${ }^{(35)}$. Autrement dit, l'heure n'est plus à la reconstruction d'une nation, mais à une internationalisation grandissante qui fait suite à la reconnaissance de l'Allemagne de l'Ouest comme nation à part entière ayant un destin similaire aux autres pays occidentaux. C'est aussi la conséquence directe de la guerre froide, qui connaît un regain avec la seconde crise de Berlin à partir de la fin des années cinquante, et de la naissance d'organismes internationaux comme l'ONU, l'Unesco ou la Communauté européenne en 1957.

\section{Le photoreportage, une esthétique étrangère sur le sol allemand}

La renaissance du photoreportage en Allemagne va accompagner ce changement. Cette redécouverte est la conséquence d'expositions de photoreporters étrangers, The Family of Man ou encore la grande exposition de l'agence Magnum au sein de la photokina de $1956^{(36)}$. The Family of Man n'est pas une exposition critique du monde mais une exposition qui tente d'en donner une approche positive comme le souhaitent alors la diplomatie américaine et l'United States Information Agency (USIA - Agence d'Information des États-Unis) qui financent son déplacement dans le monde entier ${ }^{(37)}$. Elle permet de penser le reportage hors du cadre de la presse. L'exposition Magnum a un but similaire. En montrant un reportage de chacun des membres de l'Agence, elle choisit d'exposer non pas des sujets de guerre qui avaient pourtant été à la naissance de la figure moderne du photoreporter, mais des sujets du quotidien, souvent

territoire allemand - par des photographes autochtones ou étrangers - ne rendent compte ni du destin particulier de l'Allemagne ni de la période nationale-socialiste.

33 L. Fritz Gruber, «1956, Im Auftrag der photokina: Chargesheimer », in: U. Pohlmann, Kultur, Technik und Kommerz (note 17), p. 16-19, ici p. 17.

34 « Message du Président des États-Unis », in: L. Fritz Gruber (dir.), photokina 1956, Cologne, Messeund Ausstellungs-GmbH Köln, 1956, p. 24.

35 Section historique du site officiel de la Fédération Internationale de l'Art Photographique, http://www. fiap.net/historique.php? page $=2 \&$ lang $=$.

36 «Magnum », in: L. F. Gruber (dir.), photokina 1956 (note 34), p. 147.

37 J. Szarkowski, « The Family of Man» (note 29), p. 13. 
anecdotiques $^{(38)}$. Cette mise à l'écart de la "grande histoire » au profit de l'homme va marquer les photographes allemands, déjà préparés à ce changement.

Dès la deuxième exposition qui parcourt le monde entier en 1954, la Subjektive Fotografie pâtit de son succès. De nombreux courants étrangers s'inspirent de cette photographie formelle et l'Allemagne de l'Ouest perd son statut moteur et par là sa singularité( ${ }^{(39)}$. Le photoreportage va pallier les problèmes que rencontre la Subjektive Fotografie en remplaçant la prépondérance de la forme par l'importance du sujet. La Subjektive Fotografie comme le photoreportage revendiquent tous deux un rapport à l'homme. Dans la Subjektive Fotografie, ce rapport à l'homme est directement lié au photographe créateur. Dans le photoreportage ce sont les sujets traités qui parlent de l'homme et de sa destinée universelle: portraits de célébrités, corrida par Inge Morath, « enfants sourds » de René Burri, hommes du Rhin par Cartier-Bresson donnent une image plurielle de la société des années cinquante ${ }^{(40)}$.

Ces changements se répercutent dans le champ photographique ouest-allemand. Karl Pawek va se poser en grande figure de cette nouvelle théorie photographique. Rédacteur en chef, entre 1954 et 1962, d'une revue intitulée magnum, en référence à l'Agence, il choisit de traiter des sujets universels qui donnent un aperçu de la culture contemporaine: l'homme « en soi ", le monde dans son ensemble, la société; il publie surtout, après 1958 , des numéros consacrés à la République fédérale elle-même ${ }^{(41)}$. Ce

38 Steichen accorde peu de place au reportage de guerre. Seules cinq parties sur trente-sept en comportent. Il préfère les images prises en temps de paix et les images du quotidien. Des grands photographes de la Seconde Guerre mondiale - George Rodger, Margaret Bourke-White, Robert Capa, etc. -, Steichen ne choisit pas les reportages emblématiques pris au cours de leur périple avec l'armée américaine, mais montre, a contrario, les images optimistes, celles d'une société en paix: images ethnographiques de spiritualité en Corée par Bourke-White, photographies de jeunes enfants par Lee Miller ou encore de Nubas, "dans une nudité primitive et une innocence paradisiaque » par Rodger (« in archaischer Nackheit und paradiesischer Unschuld », Viktoria Sснміdт-Lisenhoff, "Verleugnete Bilder. The Family of Man und die Shoa ", in: BAck/Schmidt-Lisenhoff (dir.), The Family of Man 1955-2001 [note 31], p. 80-99, ici p. 83). Ainsi Steichen cherche-t-il à s'éloigner de la figure du photoreporter qu'avaient construite la guerre d'Espagne et la Seconde Guerre mondiale.

39 Otto Steinert critique la « démocratisation » de la photographie expérimentale qui, pour lui, tue la Subjektive Fotografie et clôt définitivement cette période en Allemagne. Ses questionnements quant à la production expérimentale signent un enterrement en règle de la période fotoform. Les propositions du groupe, dissout en 1957, se retrouvent mis au ban de la troisième photokina qui date de 1958. Seuls Steinert et deux anciens membres - Keetman et Hajek-Halke - sont encore là sur 65 exposants. Comme il s'en explique dans un entretien avec Otto Toussaint en 1959, les travaux de fotoform sont devenus selon lui l'apanage de tous et cette vulgarisation a été fatale à la Subjektive Fotografie: "Photographier "subjectivement" est devenu une recette. " Puis il enchaîne: "S'il est vrai que l'exposition "photographie subjective" de 1951 était née de la spontanéité, il faut reconnaître que le développement ultérieur de la "subjectivité" est devenu conscient et a épuisé entièrement les moyens formels disponibles. Les expositions "photographie subjective" 2 et 3 n’ont plus rien apporté de réellement nouveau. "Sur ce constat amer, Steinert conclut par une phrase qui ressemble fortement à une question rhétorique: "La période créatrice a-t-elle touché à sa fin depuis que la masse des "utilisateurs de films" s'est adonnée à la nouvelle "recette" et en a fait une mode? ». Otto ToussaInT, "Sans retour. D'après un entretien avec Otto Steinert », Camera, mars 1959, 38 année, n³ 3, numéro spécial sur la Subjektive Fotografie 3, p. 5-6.

40 «Magnum », in: L. F. Gruber (dir.), photokina 1956 (note 34), p. 147.

41 «magnum pose des questions qui nous troublent et les ordonne en des cercles concentriques, [...] que l'on peut communément nommer par le mot-clé 'la nouvelle conception de l'homme' ", (" magnum spricht Fragen aus, die uns bewegen und ordnet sie in konzentrischen [...] Kreisen [...], die man 
parcours macroscopique de la société est porté par deux autres facteurs: la prospérité d'après-guerre et la naissance d'une nouvelle culture visuelle. Critique acerbe de la Subjektive Fotografie, Pawek explique dans un ouvrage de 1960 que : "La photographie est (devenue) le monde lui-même, la photographie est la réalité elle-même, la photographie est ce qui se passe dans cette réalité, ce qui se produit là, ce qui saigne, vit, souffre, se développe, passe là ${ }^{(42)}$. Cette adéquation entre la réalité et sa représentation est devenue primordiale. Elle permet de redéfinir les liens entre objectivité et subjectivité sur lesquels butait la photographie ouest-allemande. Pour Pawek, la prise de vue est en soi objective puisque brute et ce sont les alentours de l'acte photographique - exposition ou livre - qui permettent de donner un ordonnancement à cette réalité et qui donc font œuvre ${ }^{(43)}$. Ce point de vue, qui était déjà celui de Steichen aux États-Unis, répond aux préoccupations de la République fédérale dans cette fin des années cinquante. Cette nouvelle théorie offre des réponses aux impasses de la Subjektive Fotografie tout en profitant de ses avancées théoriques, des structures de reconnaissance mises en place dans les années d'après-guerre telles que l'édition ou les expositions dont la Subjektive Fotografie avait déjà fait usage. Le photoreporter trouve place dans un champ photographique ouest-allemand déjà constitué et prêt à l'accueillir.

Tout cela est finalement le continuum logique de ce qui eut lieu dans la première moitié des années cinquante: à la reconstitution nationale du champ fait suite une ouverture à l'international qui est le premier pas vers cet intérêt porté à la société nouvellement bâtie. Cependant, cette explosion du reportage va donner la possibilité aux photographes allemands de poser un regard critique sur leur propre société et créer un hiatus entre ce qui, jusque-là, était en adéquation: le développement de la photographie et l'évolution de la société allemande.

\section{Les photographes au cour de la critique de la reconstruction allemande}

Forte du statut international qui lui est désormais accordé et que le Traité de Rome de 1957 entérine, l'Allemagne veut oublier les traumatismes passés. Les brochures publicitaires éditées par les Länder présentent l'Allemagne comme un pays touristique, un pays de loisirs et de bien-être grâce à une reconstruction réussie et à la renaissance de

gemeinhin mit dem Schlagwort 'das neue Menschenbild’ bezeichnet »). Werner Hofmann, " Eine Zeitschrift illustriert sich selbst. Zu den ersten 4 Heften des 'Magnum’ », Forum, 1955, n 17, p. 192. Ainsi peuvent être cités les numéros Die junge Generation (1954, numéro 2), Eine Menschheit, die photographiert (1954, numéro 4), Der Mensch - Modell 1955 (1955, numéro 5), Die kleinen Menschen (1956, numéro 8), Die Situation der Frau (1958, numéro 16), Eine Lanze für den Mann (1958, numéro 20), Mensch bleibt Mensch (1959, numéro 27); Die Welt wird heiter (1955, numéro 6), Die Welt an einem Punkt (1956, numéro 9), Die Gesellschaft, in der wir leben (1957, numéro 12), Wie könnten wir leben... (1957, numéro 15); Jedem Deutschen fehlt Berlin im Kopf (1959, numéro 26), Haben die Deutschen sich verändert? (1960, numéro 29) et Bilanz der Bundesrepublik (1961, numéro 37).

42 Karl Pawek, Totale Photographie. Die Optik des neuen Realismus, Olten/Freiburg, Walter, 1960, p. 206.

43 Pour Karl Pawek, les photographies sont des mots, à partir desquels on écrit des phrases photographiques qui doivent être regardées comme des « essais optiques». Cette "'méta-photographie' devient plus intéressante que la photographie elle-même » et est la "méthode de la photographie moderne ». Voir à ce sujet: Karl PAWEK, "Le langage de la Photographie, Méthode d'agencement de cette exposition ", in: ID. (dir.), Exposition mondiale de la photographie, 555 photographies, cuvres de 264 photographes de 30 pays, sur le thème: Qu'est-ce que l'homme?, Hambourg, Henri Nannen, 1964, n. p. et Karl PAwEK, «Der neue Blick in der Photographie», magnum, 4/17 (1958), p. 6-30, ici p. 23. 
la culture allemande ${ }^{(44)}$. Certains photographes commencent cependant à émettre des critiques face à l'oubli, opéré dans les consciences, de l'histoire de leur pays. À la toute fin des années cinquante et au début des années soixante, plusieurs photographes allemands, qui avaient photographié l'immédiat après-guerre, reviennent sur le devant de la scène. Chargesheimer, Hermann Claasen et Walter Dick deviennent les nouveaux hérauts critiques du reportage politique.

Après avoir photographié les destructions de la ville de Cologne par la guerre et les bombardements alliés, Chargesheimer et Claasen photographient à nouveau la ville, dix ans après, en faisant des clichés des dernières rues de l'ancien centre-ville restées intactes, avec ses rituels ouvriers conservés, ses immeubles criblés d'impacts de balles, ses terrains vagues, toutes sortes d'images renvoyant au passé récent ${ }^{(45)}$. Pas de photos des nouveaux quartiers commerçants et bourgeois qui ont remplacé ce passé. Cette approche «nostalgique » et historique est une réponse à un livre de Chargesheimer, Cologne intime, publié l'année précédente en 1957 et dont la municipalité avait fait un livre publicitaire ${ }^{(46)}$. Chargesheimer voit dans cette récupération une trahison de taille. En 1958, il fait paraître un ouvrage sur la Ruhr, Im Ruhrgebiet, qui montre les laissés-pour-compte de la prospérité économique dans la grande région industrielle qui fut au début des années cinquante au cœur des rapprochements européens grâce au charbon et à l'acier ${ }^{(47)}$.

Chargesheimer, « Langeweil »/« Wartesaal », in: Heinrich Böll and Chargesheimer, Im Ruhrgebiet, 1958, Images 41-42. Copyright: Rheinisches Bildarchiv Köln.

44 Au titre desquels Heinrich Heidersberger, Wolfsburg, Bilder einer jungen Stadt, Munich, Bruckmann, 1963 (Réed. Berlin, Nicolaische Verlagsbuchhandlung, 2008) ou Hans Schmitt-Rost et Walter Dick, Das Bilderbuch von Köln, Cologne, Greven Verlag, 1960.

45 Voir Heinrich Böll et Chargesheimer, Unter Krahnenbäumen, Cologne, Greven Verlag, 1958 et Rolf SACHSSE, "Vom Werden und Sein eines Photographen ", in: Klaus Honnef et Rolf SACHSSE, Hermann Claasen, Landschaft, Cologne, Rheinland-Verlag GmbH, 1999, p. 17-59, ici p. 50.

46 Hans Schmitt-Rost et Chargesheimer, Cologne intime, Cologne, Greven Verlag, 1957, n. p.

47 Heinrich Böll et Chargesheimer, Im Ruhrgebiet, Cologne/Berlin, Verlag Kiepenheuer \& Witsch, 1958. 
Le livre fait scandale dès sa parution ${ }^{(48)}$. Ses vues aux tonalités noires des cités ouvrières sont en contradiction avec l'image d'un développement économique pour tous et d'une société de loisirs. Des représentants politiques dénoncent publiquement ce qu'ils considèrent comme une campagne mensongère qui dessert les intérêts de la région et de l'Allemagne ${ }^{(49)}$. Jusqu'à son dernier ouvrage en 1970, Chargesheimer gardera le regard critique sur la reconstruction née dans cette fin des années cinquante ${ }^{(50)}$.

D’autres photographes le suivent. En 1965 paraît Zeit der Ruinen (Le temps des ruines) de Walter Dick ${ }^{(51)}$. Ce livre montre la vie dans les ruines à Cologne et quelques images récentes de la ville. Publié vingt ans après les faits, à un moment où l'Allemagne est sortie de la reconstruction, il rappelle que la destruction toucha la population et modifia sa façon de vivre. Dick montre ce qui ne fut presque jamais publié: vie précaire au milieu des ruines, rapatriés avec leurs baluchons dans les rues détruites, enfants volant quelques briquettes du chargement d'un camion, misère, marché noir, baraquement de fortune... Sans présenter d'images de guerre ou d'occupation, l'ouvrage rappelle aux Allemands que la période faste des années cinquante s'est construite sur les ruines de l'Histoire.

Face à ce monde définitivement perdu à cause du nazisme puis d'une reconstruction rapide qui a cherché à tout gommer, photographes et écrivains ont un regard proche et viennent à collaborer tel Heinrich Böll à l'édition de nombreux ouvrages de photographie dont ceux de Chargesheimer ${ }^{(52)}$. Comme les écrivains du Groupe 47, les photographes se forgent un regard critique à l'égard de leurs dirigeants politiques et du tournant pris par l'Allemagne de l'Ouest dans la quinzaine d'années qui suit la capitulation. Cette société bourgeoise et conservatrice qui s'est mise en place, Chargesheimer la caricature librement lorsqu'il fait en 1957 le portrait de Konrad Adenauer, alors âgé de 81 ans, en vieillard ridicule, alors même que celui-ci brigue un nouveau mandat ${ }^{(53)}$. L'image diffusée le 11 septembre 1957 en une du Spiegel fait scandale. On parle de diffamation du plus haut représentant du gouvernement ${ }^{(54)}$. Le photographe refuse ici toute participation à une « histoire sublimée ».

48 Voir: Sigrid Schneider, “'Solche Darstellungen akzeptieren wir nicht'. Zur Rezeption des Bildbands Im Ruhrgebiet von Heinrich Böll und Chargesheimer ", in: Bodo von Dewitz (dir.), Chargesheimer. Bohemien aus Köln 1924-1971, Cologne, Museum Ludwig/Greven Verlag, 2007, p. 241-247.

49 Le maire de Bochum, comme le rapporte Der Spiegel, se sent " totalement trompé " (" restlos getäuscht») dans ses attentes, il ne peut « utiliser le livre [...] à des fins de publicité municipale » ("das Buch [...] für Zwecke der Stadtwerbung verwenden zu können »). Der Spiegel, 21.1.1959 (Succession Chargesheimer, Museum Ludwig, Cologne).

50 Voirson dernier ouvrage:Chargesheimer, Köln5Uhr30, Cologne, VerlagM. DuMontSchauberg, 1970.

51 Hans Sснмітт-Rost et Walter Dick, Zeit der Ruinen, Köln am Ende der Diktatur, Cologne, Kiepenheuer \& Witsch, 1965.

52 Heinrich Böll se charge aussi de la préface du livre de Walter Dick dans laquelle il décrit: «la poussière et le silence " ("der Staub und die Stille») matérialisés par les images d'architectures brisées, et «la vie nue » (« das nackte Leben »). Heinrich Böll, « Heimat und keine », in: Schmitt-Rost/ Dick, Zeit der Ruinen (note 51), p. VII-XI, ici p. X.

53 Il faut dire que Der Spiegel la publie l'année des élections, que le journal affiche un parti pris pro-opposition et que les instituts de sondages donnent encore le SPD en tête. Par ses attaques ciblées envers le chancelier, Der Spiegel contribue à personnaliser le débat et à le dépolitiser " pour transformer les élections en plébiscite ». Alfred Grosser, "Le plébiscite du 15 septembre 1957 ", Revue française de science politique, 7/4 (1957), p. 839-864, ici p. 843 et 854 .

54 Voir Bodo von Dewitz (dir.), « Chargesheimer. Bohemien aus Köln », in: ID. (dir.), Chargesheimer (note 48 ), p. 9-17, ici p. 11. 
L'indépendance que retrouvent ces photographes dans leur critique de la société va peser sur le champ photographique. Ils refusent les grandes théories et préfèrent construire eux-mêmes un discours autour de leur production artistique par la publication de livres qui ne sont pas l'objet de commandes. Ainsi reprennent-ils les rôles que s'était octroyé Otto Steinert avec la Subjektive Fotografie: ceux de praticien, théoricien, commissaire d'exposition et éditeur. Mais contrairement à celui-ci, ces photographes ne cherchent plus à construire ou reconstruire un champ, à baliser les utilisations du médium ou à lui offrir une épistémologie. Ils travaillent à leur échelle et pour leur propre production. Ne cherchant plus à développer de théories d'ensemble comme dans les années d'après-guerre et revendiquant une autonomie qui tendait à disparaître, ces photographes sont à l'origine de l'éclatement du champ photographique uni de ces années-là. Une nouvelle figure du photographe voit le jour: un photographe qui emprunte ses sujets au photoreportage et ses moyens de diffusion à la photographie artistique, un photographe qui revendique l'indépendance de son médium, autant qu'un rôle critique et une place dans l'espace social. L'émergence, dans les années qui suivent, de figures comme les époux Becher ne peut se comprendre que dans ce contexte. Mais, s'il a accouché de figures-phares, ce regard critique porté sur l'Allemagne a démantelé dans le même temps le champ photographique uni de la reconstruction.

Ce regard critique de l'après-1955 est un retour indirect aux propos des photographes allemands formulés dans les toutes premières années de l'après-guerre, et qui dénonçaient la destruction des villes allemandes comme issue de la sécularisation dans un Occident chrétien ${ }^{(55)}$ et de la montée du national-socialisme ${ }^{(56)}$. En 1949, le discours critique concernait le passé. À la fin des années cinquante, il se pose sur un présent qui tente de faire oublier une histoire que l'on veut effacer et qui cherche à dissimuler les difficultés que rencontre la nouvelle République fédérale d'Allemagne. Ce retour à la figure du photographe engagé n'a été possible que grâce aux dix ans qui ont précédé et à la reconstruction qui s'est opérée. Dans cet intervalle, la photographie allemande a conquis une indépendance et une reconnaissance grâce à une unité du champ qui a conduit à monter des expositions, élaborer des théories et publier des magazines spécialisés, autant de vecteurs que les photographes de la deuxième moitié des années cinquante vont pouvoir utiliser. La construction de ce champ unifié et autonome qui épousa le destin allemand peut alors être qualifiée de détour salvateur et nécessaire, qui a permis à la photographie allemande d'asseoir sa légitimité, de s'imposer comme un champ culturel, mais surtout de développer des enjeux qui lui étaient propres et restaient indépendants des autres champs artistiques. Ainsi, l'adéquation du champ

55 Pour Hermann Claasen, par exemple, c'est la disparition progressive de la foi en Occident et particulièrement dans le Reich allemand qui est à l'origine de ce qui a pu se produire en Allemagne. Le national-socialisme et la Seconde Guerre mondiale doivent, pour le photographe, être analysés comme les conséquences d'une perte générale de la croyance en Dieu.

56 Au nombre desquels Hermann ClAASEN, Gesang im Feuerofen, Köln - Überreste einer alten deutschen Stadt (note 12); Kurt SchaArschuch, Bilddokument Dresden. 1933-1945, Dresde, Dresdner StadtVerlag, 1945, et deux ouvrages posthumes: Winfried Ranke, August Sander, Die Zerstörung Kölns, Photographien 1945-46. Mit einem Text von Heinrich Böll, Munich, Schirmer/Mosel, 1985; Ludger Derenthal et Ulrich Pohlmann (dir.), Herbert List - Memento 1945, Münchner Ruinen, Munich, Schirmer/Mosel, 1995. 
photographique à l'histoire de la jeune république durant les dix premières années n'a pas été synonyme de dépendance, contrairement à ce qui s'était passé pendant la guerre avec les accointances propagandistes, mais lui a permis de conquérir sa liberté ${ }^{(57)}$.

\title{
Résumé
}

Dans les premières années de l'après-guerre, le champ de la photographie se construit dans une accointance directe au destin de l'Allemagne. De l'occupation où l'utilisation du médium est avant tout politique au développement économique des années cinquante, tout dans le domaine photographique semble en faire le miroir à petite échelle de la construction de la République fédérale. Pourtant, à partir de 1955, la photographie vient à critiquer, voire dénigrer, une bonne part des projets qu'elle avait jusque-là défendus, démontrant l'autonomie progressive que n'aura de cesse de s'octroyer le médium. La réflexion menée dans cet article s'appuie sur les composantes économiques et théoriques du champ photographique en regard des productions artistiques, sans faire l'impasse sur les livres illustrés (Chargesheimer, Hermann Claasen, Walter Dick) et les expositions importantes de l'époque dont celles de la photokina de Cologne.

\section{Zusammenfassung}

In den ersten Nachkriegsjahren bildet sich die Fotografie in einem direkten Bezug zum Werdegang Deutschlands heraus. Der gesamte Bereich der Fotografie macht aus dem Medium von der Besatzungszeit an, wo die Fotografie vor allem politisch genutzt wird, über die ökonomische Entwicklung bis hin zur Blüte Anfang der 50er Jahre, eine Spiegelung kleineren Maßstabs des deutschen Wiederaufbaus.Unsere Überlegungen beziehen sich auf den Bereich im Zusammenhang mit den ökonomischen und theoretischen Komponenten im Blick auf die künstlerischen Produktionen. Wir untersuchen anhand von Bildbänden (Chargesheimer, Hermann Claasen, Walter Dick), bedeutender Ausstellungen jener Zeit - darunter die photokina in Köln

\begin{abstract}
In the first years after World War II, the photographic field follows the West German destiny. During the occupation, the utilization of this medium is first a political one. Until the economic development, the photographic field seems to be the perfect reflection of the building of the GDR. Though, from 1955, photography begins to critic an important part of what it was fighting for. The medium becomes more independant. This articles try to understand this changement by examinate the economical and theorical components of the photographic field and the illustrate books (Chargesheimer, Hermann Claasen, Walter Dick) and the essential exhibitions like the photokina in Cologne.
\end{abstract}

57 L'auteur tient à remercier les musées et institutions qui ont bien voulu mettre à sa disposition les reproductions illustrant l'article. 